\title{
Research for motion prediction system of multi-axis motion controller
}

\author{
He Yin ${ }^{1, *}$, Quan-Gang Wen ${ }^{1}$, Zhi-Gang Hao ${ }^{1}$ and Ting-Ting $\mathrm{Li}^{2, *}$ \\ ${ }^{1}$ Computer Department, Zhuhai College of Jilin University, Zhuhai, Guang Dong, China \\ ${ }^{2}$ Basic teaching Department, Zhuhai College of Jilin University Zhuhai \\ E-mail:yinhe702@163.com,wen_sir_125@163.com,haozhigang001@sina.com, \\ 123058904@qq.com \\ *Correspondind author
}

\begin{abstract}
In this paper, the problems of multi-axis motion controller were analyzed, and then a predictive control method of controlling stepper motor speed for execute $\mathrm{G}$ code file was proposed, the principle of the method being analyzed and deduced. The method was emulated in Matlab, being analyzed and compared with actual data ,the original $\mathrm{T}$ shape acceleration and deceleration algorithm.

Keywords: Multi-axis Motion Controller; G Code; Predictive Control; $\mathrm{T}$ shape Acceleration and Deceleration Algorithm.
\end{abstract}

\section{Foreword}

In order to ensure the smooth operation of $\mathrm{CNC}$ equipment (CNC), we need a good speed control method of the servo motor start, stop, acceleration and deceleration process as far as possible to avoid the occurrence of shock, loss of step, shock or super phenomenon. Therefore, servo motor acceleration and deceleration control is one of the difficulties of servo system , $\mathrm{T}$ shaped algorithm is easy to implement, but in the middle of two times same direction $\mathrm{T}$ shape acceleration and deceleration motion in a straight line, slowed down, stop, next acceleration will impact on the equipment.

In view of the above problems, this paper presents an adaptive forward interpolation method, can achieve twice to $\mathrm{T}$ shape acceleration and deceleration motion in a straight line between the motor does not stop, and in the initial velocity of the next movement entered a motion in a straight line, thus greatly reduce the impact of motor. Algorithm simulation in the MATLAB environment, connecting with the actual examples, analyzed and compared with the original T-shaped acceleration and deceleration algorithm. Then the algorithm is applied to the multi-axis control system, and it has achieved good application results. 
Arrange the following chapters: In the second part, the platform of multiaxis motion control system is introduced. the $\mathrm{G}$ code implementation are analyzed. In the third part, original T-shaped acceleration and deceleration algorithm and improved algorithm are deduced,and emulated in the MATLAB environment. In the fourth part,the improved motion control method is realized in the motion control platform. The fifth part is the conclusion.

\section{Implementation of NC Code Compiler and Motion Control}

The motion controller is the core component of the system motion control, It is extensively used in many works[1]. The hardware of embedded multi-axis motion controller consists of two parts: ARM main control board and FPGA motion control board, two pieces of circuit board connected with FSMC bus interface. ARM main control board is arranged with abundant memory resources and universal device interface, responsible for the completion of the task management, data receiving and processing, NC code compiler, input and output, control external equipment; FPGA motion control board is responsible for the real-time interpolation, concurrent veins impulse control motor speed, complete the trajectory control.

\subsection{NC code compiler introduction}

In the software design of motion controller, embedded real-time operating system on RT thread is transplanted to the ARM processor, the EMC2 (LinuxCNC) $\mathrm{G}$ code parser ported to operating system, analysis of the $\mathrm{G}$ code file, and $\mathrm{G}$ code instruction information from assignment to the FPGA control board, to realize the interpolation movement of motor.

\subsection{G code file execution process}

NC code compiler is a CNC system of three core part of, its main function is to user programs, including the trajectory information, speed, size, and auxiliary function information, translation for motion control module can handle the format.

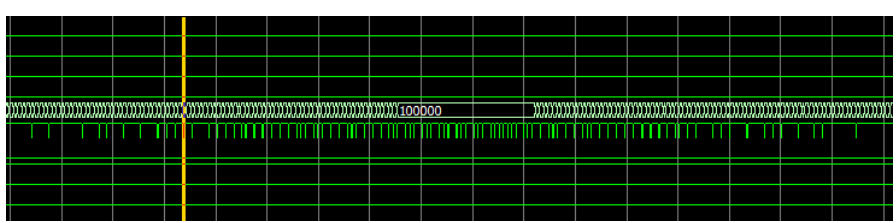


Fig. 1 Once movement the FPGA pulse instructions issued to the motor

There are two mainly processing methods of NC code in the NC system: Line by line realization method and compile method. Line by line realization method is read one line of code and then processing, it is simple to achieve this method [2]. But the two movement has no relation, the efficiency is low, see Figure 1.

\section{Improvement principle and Simulation of acceleration and deceleration algorithm}

\subsection{T-shaped acceleration and deceleration algorithm}

In order to facilitate comparison, we use the same parameters in the case of the $\mathrm{T}$ - shaped acceleration and deceleration algorithm. The complete $\mathrm{T}$ - shaped acceleration and deceleration algorithm is composed of three stages: acceleration, Eq. (1), uniform speed ,Eq. (2)and deceleration ,Eq. (3).

$$
\begin{gathered}
V_{\mathrm{t}}=V_{0}+a \times \mathrm{t} \\
V_{\mathrm{t}}=V_{\max } \\
V_{\mathrm{t}}=V_{\text {max }}-a \times \mathrm{t}
\end{gathered}
$$

$V_{0}$ is the initial velocity in the equation, $V_{\mathrm{t}}$ is the velocity of a certain moment, $a$ is the acceleration, $V_{\max }$ is the maximum velocity, $\mathrm{t}$ is the acceleration and deceleration time, According to the equation(1)-(3), We can get the simulation of $\mathrm{T}$ - shaped acceleration and deceleration algorithm, see Figure 2 .

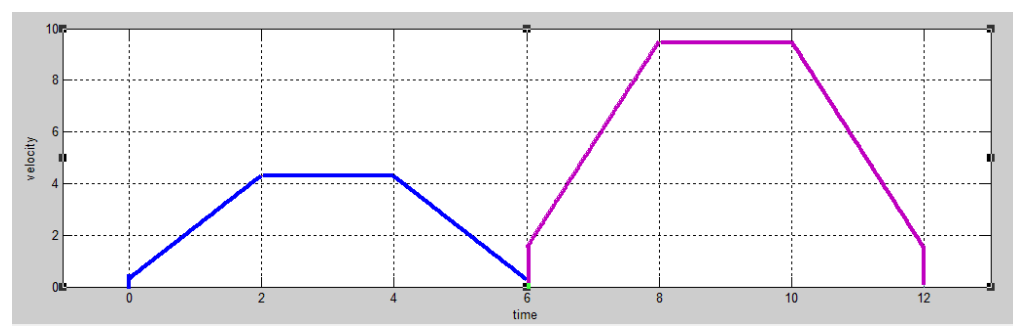

Fig. 2 Simulation of two time continuous independent motion $\mathrm{T}$ shape acceleration and deceleration algorithm in MATLAB 
From the graph, we know that the initial velocity of the $\mathrm{T}$ shape acceleration and deceleration algorithm is changed to the maximum speed with the maximum acceleration. The advantage is that the efficiency of the acceleration phase and deceleration phase is the highest, however, in the two continuous movement, the motor must have the same speed as the initial velocity of the next movement during deceleration. If it could be at this time, move to the specified position with this speed, rather than wait until the motor stops, then restart from zero acceleration, can reduce the impact on the motor.

\subsection{Principle of prediction algorithm}

Referring to the speed formula of $\mathrm{T}$ - shaped acceleration and deceleration algorithm, this algorithm takes into account the two motion of the same direction, acceleration and deceleration of the motor is always the largest and same. When the speed of the previous movement slows down to the initial velocity of the next movement, Stop slowing down, get the equation (4)-(6):

$$
\begin{gathered}
V_{t}=V_{\max 1}-a_{\max } \times \mathrm{t}_{1} \\
V_{t}=V_{0}^{\prime} \\
V_{0}^{\prime}=V_{\max 2}-a_{\max } \times \mathrm{t}_{2}
\end{gathered}
$$

$V_{\mathrm{t}}$ is the velocity of a certain moment, $V_{\max 1}$ is the maximum speed of the previous movement, $a_{\max }$ is the maximum acceleration, $V_{0}^{\prime}$ is the initial velocity of the next movement, $t_{1}$ is the time of previous movement deceleration, $t_{2}$ is the time of next movement acceleration, $t$ and $t_{2}$ is the rule acceleration and deceleration time, $t_{1}{ }_{1}$ is the time of constant speed. We know the first movement deceleration time according to the equation (7).

$$
\begin{gathered}
\mathrm{t}_{1}=\mathrm{t}_{2}+\frac{V_{\max 2}-V_{\max 1}}{a_{\max }} \\
\mathrm{t}_{1}^{\prime}=\mathrm{t}-\mathrm{t}_{1}
\end{gathered}
$$




$$
s^{\prime}=V_{0}^{\prime} \times \mathrm{t}_{1}^{\prime}
$$

From equation (4)- (9),we know that ,through speed optimization, when the speed of the previous movement slows down to the initial velocity of the next movement, the motor to move to the specified location at constant speed, and the next movement accelerate from this speed, it reduce the impact on the motor.

Through the above analysis, we have simulated the acceleration and deceleration algorithm in MATLAB environment, see Figure 3.

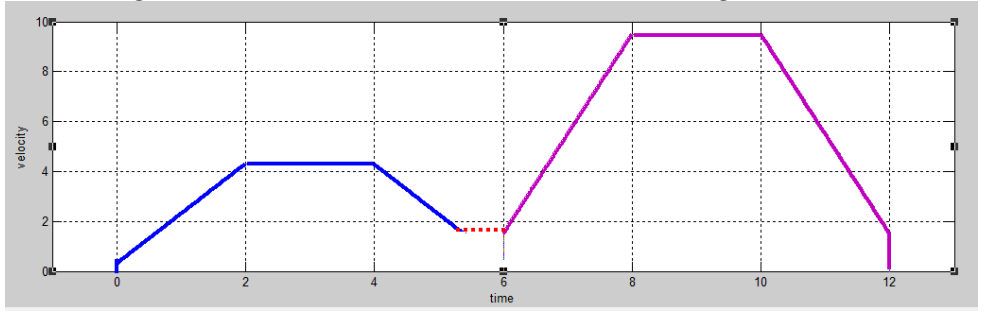

Fig. 3 After optimization the simulation of two time continuous independent motion $\mathrm{T}$ shape acceleration and deceleration algorithm in MATLAB

\section{Implementation of predictive control algorithm in motion control system}

\subsection{The implementation process of the original system $G$ code}

An example of $\mathrm{G}$ code program:

G01 X51 F25;

After the $\mathrm{G}$ code compiler, the position information and speed information were assigned to the two functions respectively:

1.void STRAIGHT_FEED(double x, double y, double z);

2.void SET_FEED_RATE(double rate);

Functions contains the execution function of FPGA:

1. pulse $(0 \times 1, \mathrm{x} 1-\mathrm{x} 0)$;

2. $\operatorname{speed}(0 \times 1$, rate $)$;

\subsection{Program improvement of $G$ code compiler}

An example of two continuous action $\mathrm{G}$ code program :
1. G01 X51 F25;
2. G01 X102 F30; 
Increase judgment in function:void STRAIGHT_FEED(double x, double y, double $\mathrm{z}$ ), If the two actions is same coaxial and direction, slow down in the first action to the initial speed $\left(V_{0}^{\prime}\right)$ of the second action, Executive function: speed $\left(0 x 1, V_{0}^{\prime}\right)$,keep the speed at this value, then execute the next set of FPGA instructions, make the motor to continue to accelerate on this speed, see Figure 4.

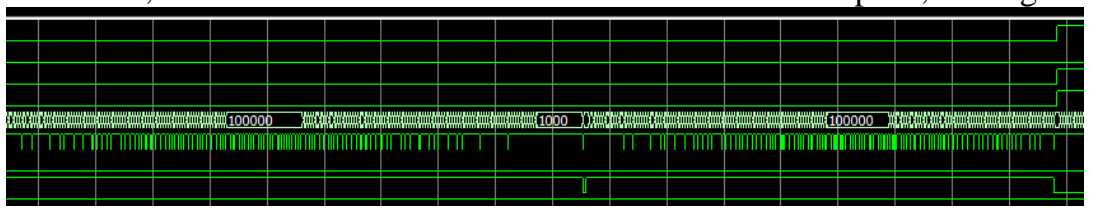

Fig. 4 Two successive movement the FPGA pulse instructions issued to the motor

\section{Summary}

In this paper, the problems of multi-axis motion controller were analyzed, and then a predictive control method of controlling stepper motor speed for execute $\mathrm{G}$ code file were proposed, then the principle of the method is analyzed and deduced. The method were emulated in Matlab and compared and analyzed with actual data and the original $\mathrm{T}$ shape acceleration and deceleration algorithm.

\section{References}

1. Lushan Liu, Design and research of embedded four axis motion controller, Master's thesis, Guangdong University of Technology (2007).

2. LI Shan-feng, Liu Jing-meng, Chen Bai-cheng,Xu Dong, Design of an embedded NC code compiler, Modular Machine Tool \& Automatic Manufacturing Technique,.2012.2, 1001-2265(2012)02.

3. Wu Jibin, Research on the key algorithms of motion control and the implementation of embedded system, Master's thesis, South China University of Technology (2010).

4. Liu Jiankang, The hardware design of Embedded CNC system based on ARM_FPGA, Harbin Institute of Technology (2007). 